\title{
An efficient 3D point cloud data denoising algorithm for ship block visual measurement
}

\author{
Liu Tao ${ }^{1, *}$, Du Hongwang ${ }^{1}$ \\ ${ }^{1}$ College of Automation, Harbin Engineering University, Harbin 150001, China
}

\begin{abstract}
Keywords: ship block measurement; point cloud data; denoising; least square fitting
Abstract. For the 3D point cloud data aquired by laser scanning measurement of ship block, an efficient denoising algorithm based on image and normal vector threshold judgement is proposed. Firstly, large scale noise points are eliminated using global threshold judgement based image, then Kuwahara filter algorithm is used for data smoothing and a denoising algorithm based on normal vector threshold judgement is proposed to eliminate noises point excluding ship manufacture sections. The experiment result demonstrates that not only the proposed denoising algorithm keeps key data points but also avoids bluring point cloud boundary and eliminates noise points effectively.
\end{abstract}

\section{Introduction}

As manufacturing sections of ship block are composed of a lot of steel plates, 3D point cloud data obtained by structured light vision measurement sensor is not a geometry distribution to some extent, which is scattered and disordered. A few noise point cloud data excluding the ship segmentation during vision measurement procedure will be obtained simultaneously. So point cloud data denoising is an essential alogorithm in reverse engineering; point cloud denoising processing will directly affect the quality of subsequent 3D model reconstruction, which significantly affects the analysis and manufacture of results using the $3 \mathrm{D}$ model.

Common method of data smoothing and denoising are filtering technology in digital image processing, which are divided into space domain and frequency domain filtering. In recent years, many scholars have put forward various point cloud denoising algorithms ${ }^{[1-4]}$. These methods are generally facing the challenge both to reduce noise and to ensure the point cloud important feature information unaffected. According to the characteristics of different 3D objects, different point cloud denoising algorithms need to be putted forward. During the point cloud data processing of ship sections, some useless outlier points are needed to removed, and the background points need processing in order to the point cloud feature extraction subsequently. According to the point cloud data generated of ship sections visual measurement, a point cloud denoising algorithm is putted forward. Firstly based on image the large areas background noise points are removed, then using Kuwahara ${ }^{[5]}$ filtering algorithm for data smoothing, finally a denoising algorithm based on normal vector threshold is proposed to eliminate noise data outside of manufacturing section. The algorithm firstly calculate $K$ neighborhood point, then calculate plane tangent of the least square fitting for $K$ neighborhood point, finaly use the angle of measuring point with tangent plane normal vector and the setting plane vector direction to distinguish whether to remove the measuring points.

\section{Point cloud data denoising based on image}

The feature point cloud of ship block manufacturing section obtained by digital measurement is basically in a section, so data depth has little difference, and the target data depth value basically than the noise data is smaller. Using the above information, based on the concept of digital image processing, the obtained data points are taking as image data, the measurement data points depth value as the pixel gray values in the image. Using the depth value distribution of the statistical point cloud data in histogram image processing, target data depth range ranges were counted, in order to remove large area noise point.The global thresholding method is as follow:

Step1 Choose an initial estimate $T_{0}$ for $T$ in the middle of the maximum and minimum value.

Step2 Uses $T$ to segmentation point cloud data. Achieve two sets of point cloud data: $G_{1}$ composed of all $z \geq T$ pixel, $G_{2}$ composed of all $z<T$ pixel.

Step3 Calculates the average $z$ with point cloud data $\mu_{1}$ and $\mu_{2}$ within the range of values $G_{1}$ and $G_{2}$.

Step4 Calculates a new threshold: $T=\frac{1}{2}\left(\mu_{1}+\mu_{2}\right)$

Step5 Repeat steps 2 through 4 successive iteration, until the $T$ value is smaller than the pre specified parameters $T_{0}$.

\footnotetext{
"Corresponding author: hrbeu411jys@163.com
} 
For point cloud data obtained by laser scanning measurement, common filtering algorithms cannot remove noise and preserve edge also has good effect. In order to obtain the boundary characteristics accurately, which can eliminate the noise but also to ensure the edge, corner feature is not smooth, using the Kuwahara filtering algorithm $^{[5] .}$ Kuwahara filtering algorithm is based on the depth image which is the famous boundary preserving smoothing method.

\section{Denoising method of point cloud data based on normal vector threshold judgement}

\subsection{The $K$ neighbourhood calculation}

The three-dimensional point cloud of block visual measurement, have data redundancy, the existence of incomplete data and point cloud data with unknown topology. The establishment of geometric topology relationship between measuring point cloud, is beneficial to reduce the search range data and improve the dense scattered point cloud modeling speed. The common establishment method of data point cloud topological relationship is space grid method, such as proposed 3D scattered data nearest neighbor search algorithm ${ }^{[6]}$. The ship section measuring point cloud data processing used a fast searching large data sets $K$ nearest neighbor spatial sphere algorithm ${ }^{[7]}$, which is easy to realize and can quickly detect the point nearest neighbor.

\subsection{Calculation of tangent plane based on least square fitting}

Normal vector and curvature are the basic characteristics of surface, and are important bases of surface feature recognition. The normal vector can be determined by the tangent plane of the point ${ }^{[8]}$. Manufacturing section model of ship sections were positioned substantially in the plane, so the plane can be obtained by using least squares fitting.

For any point $P$ in space, find its $K$ neighbor and include the point denoted $\operatorname{Nbhd}(P)$, ensure the minimum square of the distance from the $K$ neighborhood of the point to the tangent plane, the normal vector can be calculated, and calculate the point approximation tangent plane by plane equation.

Set $\operatorname{Nbhd}(P)$ as approximation of planar equation for a:

$$
A x+B y+C z+D=0
$$

The unit normal vector plane is $N(A, B, C)$, then the distance formula of $\operatorname{Nbhd}(P)$ point and plane $P_{i}\left(x_{i}, y_{i}, z_{i}\right)$ can be expressed as follows:

$$
D_{i}=\left|A x_{i}+B y_{i}+C z_{i}+D\right|
$$

$K$ neighborhood point set is $P_{i}(i=1,2,3, \cdots, k)$, by the distance minimum square of these points and the least square plane, the objective function is established:

$$
F(A, B, C, D)=\sum_{i=1}^{k}\left(A x_{i}+B y_{i}+C z_{i}+D\right)^{2}
$$

To make $F$ minimum, dealing with the $F$ derivative, which is equal to zero, get:

$$
D=-\left(A x_{c}+B y_{c}+C z_{c}\right)
$$

Among: $x_{c}=\frac{1}{k} \sum_{i=1}^{k} x_{i}, \quad y_{c}=\frac{1}{k} \sum_{i=1}^{k} y_{i}, \quad z_{c}=\frac{1}{k} \sum_{i=1}^{k} z_{i}$ 。

$\left(x_{c}, y_{c}, z_{c}\right)$ is the center coordinates of point set $P_{i}$.

Eq (3) has:

$$
F(A, B, C, D)=\sum_{i=1}^{k}\left[A\left(x_{i}-x_{c}\right)+B\left(y_{i}-y_{c}\right)+C\left(z_{i}-z_{c}\right)\right]^{2}
$$

The Eq (5) derivative is:

$$
\left[\begin{array}{lll}
a_{00} & a_{01} & a_{02} \\
a_{10} & a_{11} & a_{12} \\
a_{20} & a_{21} & a_{22}
\end{array}\right]\left[\begin{array}{l}
A \\
B \\
C
\end{array}\right]=0
$$

Introduce standard constraint condition in the plane normal vector $A^{2}+B^{2}+C^{2}=1$ The problem is under the constraint condition of extremum. The constraint conditions are:

$$
G(A, B, C)=A^{2}+B^{2}+C^{2}-1
$$

By Lagrange multiplication:

$$
\begin{gathered}
\nabla F(A, B, C)=\lambda \nabla G(A, B, C) \\
\left\{\begin{array}{lll}
{\left[\begin{array}{lll}
a_{00} & a_{01} & a_{02} \\
a_{10} & a_{11} & a_{12} \\
a_{20} & a_{21} & a_{22}
\end{array}\right]\left[\begin{array}{l}
A \\
B \\
C
\end{array}\right]=\lambda\left[\begin{array}{l}
A \\
B \\
C
\end{array}\right]} \\
A^{2}+B^{2}+C^{2}=1
\end{array}\right.
\end{gathered}
$$

The expansion is:

$$
\left\{\begin{array}{l}
\sum A\left(x_{i}-x_{c}\right)\left[A\left(x_{i}-x_{c}\right)+B\left(y_{i}-y_{c}\right)+C\left(z_{i}-z_{c}\right)\right]=\lambda A^{2} \\
\sum B\left(y_{i}-y_{c}\right)\left[A\left(x_{i}-x_{c}\right)+B\left(y_{i}-y_{c}\right)+C\left(z_{i}-z_{c}\right)\right]=\lambda B^{2} \\
\sum C\left(z_{i}-z_{c}\right)\left[A\left(x_{i}-x_{c}\right)+B\left(y_{i}-y_{c}\right)+C\left(z_{i}-z_{c}\right)\right]=\lambda C^{2}
\end{array}\right.
$$

The sum of all kinds:

$$
\sum\left[A\left(x_{i}-x_{c}\right)+B\left(y_{i}-y_{c}\right)+C\left(z_{i}-z_{c}\right)\right]=\lambda
$$

According to the center coordinates and the above method the tangent plane equation can be determined.

\subsection{Data processing based on normal vector threshold judgement}

Setting normal vector threshold on feature regions, greater than the threshold point cloud grids are delete, so we can get a single value plane (space plane projection direction does not coincide). Set reference plane normal vector $n$ of hip manufacturing section, each measuring point $x_{i}$ nearby is calculated and the close points are fitted as the constraint tangent plane $P\left(x_{i}\right)$ of the point nearby. Keeping minimum distance square sum of the $K$ neighborhood to tangent plane, calculate the normal vector $n_{i}$ of the tangent plane, use $\alpha_{i}$ to show normal s vector angle of the measuring threshold. If $v_{1}>\alpha_{i}>v_{2}$ is present, measuring 
point is removed.

By the tangent plane projection, 2D projection point set can beobtained. The coordinates of the point are $\left(x^{\prime}, y^{\prime}, z^{\prime}\right)$. The projection Coordinates of points in the plane are $(x, y, z)$. The line is through a point $\left(x^{\prime}, y^{\prime}, z^{\prime}\right)$ and direction vector $N(A, B, C)$. The $\mathrm{Eq}(18)$ is obtained and $\mathrm{By}$ the $\mathrm{Eq}(1)$ and $\mathrm{Eq}(7)$ the simultaneous solution of the projection point coordinates can be obtained

$$
\frac{x-x^{\prime}}{A}=\frac{y-y^{\prime}}{B}=\frac{z-z^{\prime}}{C}=k
$$

After finding the tangent plane, through coordinate transformation to the coordinates of the points into the two-dimensional coordinates, you can consider only the two coordinate value without considering the third direction coordinate values, to simplify the problem. Of course, change the coordinates of the projection plane do similarly applied to all spatial data sets, to maintain data consistency and projection plane.

\section{Experiments}

The obtained point cloud data of ship block visual measured in different parts of the model is denoising, and the experimental results are analyzed. Figure 1(a) and (c) show point cloud obtained of ship model frame scan. The scanned point cloud includes the local data points and large areas noise point block which can be seen, so the denoising is very necessary for data processing. Figure 1 (b) and (d) show the experimental results for denoising the data point cloud by using this proposed algorithm. Figure 2 (a) shows the plane graph of point cloud data denosing of ship block model, Fig. 2 (b) is shown amplification maps in Figure 2 (a) arrow site. Through the denoising point cloud data the large background noise are effectively eliminated.

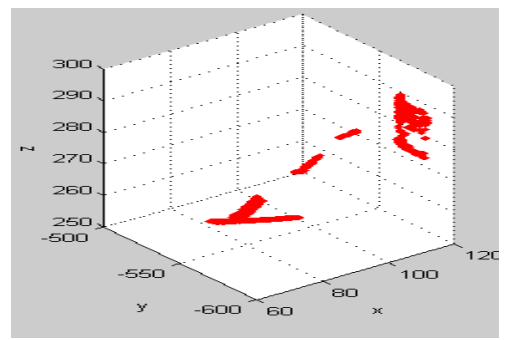

(a) the rib cage point cloud data measured

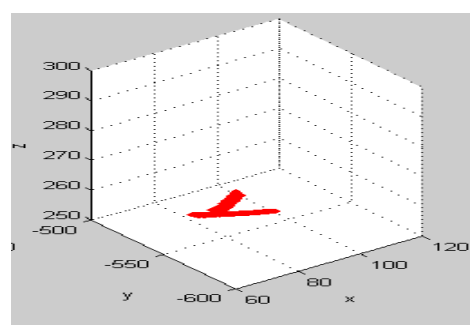

(b) the rib cage point cloud data after denoising

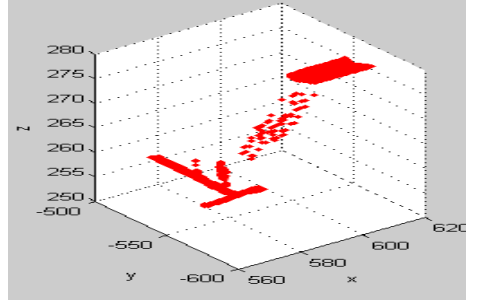

(c) T type of rib point cloud data measured

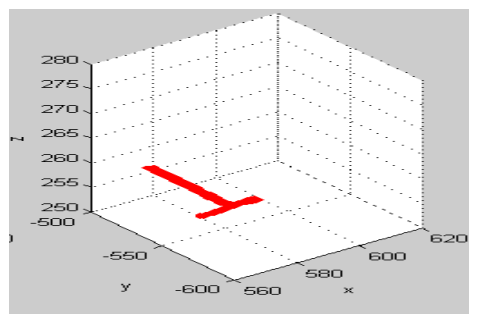

(d) the rib cage point cloud data after denoising

Fig.1 Schematic diagram of denoising for the point cloud of ship block measurement

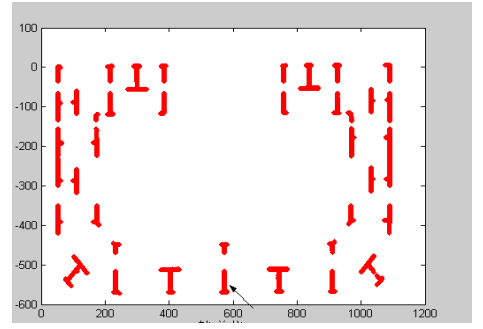

(a) the plane graph of point cloud data denosing

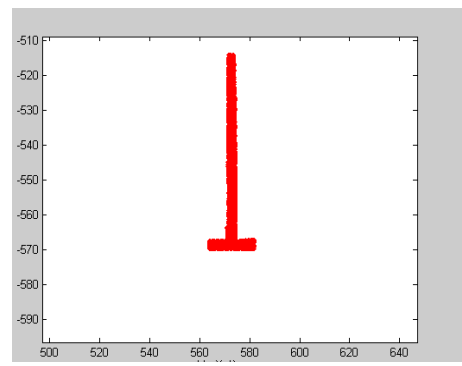

(b) the point cloud data locally amplified of ship block model

Fig. 2 Schematic diagram of de-noising for point cloud of ship block model

\section{Conclusions}

For 3D point cloud data of the ship block model vision measurement, the efficient point cloud data denoising algorithm has been researched deeply. The algorithm firstly calculate $K$ neighborhood point, then calculate tangent plane tangent of the least square fitting for $K$ neighborhood point, finaly use the angle of measuring point with tangent plane normal vector and the tangent plane vector direction to distinguish whether to remove the measuring points. The experimental results validate the effectiveness of the proposed algorithms. 


\section{Acknowledgements}

This project was financially supported by the Heilongjiang Province Natural Science Foundation (F201342), and National Natural Science Foundation of China (51409059), Heilongjiang Province Postdoctoral Research Foundation(LBH-Q16066) and China Scholarship Council .

\section{References}

[1] Kai W, Dieter R, Dirk S. An approach to computer-aided quality control based on 3D coordinate metrology. Journal of Materials Processing Technology. 2000,107: 96-110P

[2] Li jiangxiong, Ke yinglin . Reverse engineering of complex surface based on feature. CHINESE JOURNAL OF MECHANICAL ENGINEERING. 2000,36(5):18-21P

[3] ZHANG Xue-chang etc. Study on the Choice of Disciplinal Function During Point Cloud Smoothing Based on Anisotropic Heat Diffusion. JOURNAL OF SHANGHAI JIAOTONG UNIVERSITY. 2008,42(10):1687-1691

[4] Liu dafeng etc. Scattered point cloud to research and implementation of noise algorithm. Journal of Southeast University(Natural Science Edition). 2007,37(6):1108-1112

[5] M.Kuwahara, K.Hachimura, S.Eiho, M.Kinoshita. Digital Processing of Bionedical Images. 1976

[6] Xiong bangshu etc. Three dimensional scattered data $\mathrm{K}$ nearest neighbor search algorithm. Journal of Computer Aided Design \& Computer Graphics. 2004,16(7):909-912

[7] Wei wei etc. A quick search of the massive data sets K nearest neighbor spatial sphere algorithm. Acta Aeronautica Et Astronautica Sinica. 2006,27(5):944-948

[8] F.P Ferrie, J.Lagarde, P.Whaite. Darboux frames, snakes, and super- quadrics: geometry from the bottom up. IEEE Transaction s on Pattern Analysis and Machine Intelligence. 1993,15(8):771- 783 